\title{
UNA PROPUESTA EDUCATIVA PARA ATENDER LA DIVERSIDAD EN EL CONTEXTO DE AULA
}

M.Ed. Rosario Agüero Agüero ${ }^{1}$

\section{RESUMEN}

En la actualidad, los centros educativos tienen el gran reto de brindar a sus estudiantes una educación de calidad y principalmente una educación para todos. El artículo tiene la finalidad de promover el respeto por las diferencias individuales y el de atender la diversidad en los contextos escolares. El proceso diagnóstico realizado determinó que las y los estudiantes muestran actitudes de rechazo e irrespeto hacia las diferencias individuales vistas desde cualquier aspecto.

Se consultaron autores principalmente a Meléndez (2002), Parcerisa (2000), Olivé (1999) y Aguilar (2000), quienes brindan algunos lineamientos y consideraciones a utilizar en un centro educativo que busque atender la diversidad estudiantil. Los mismos autores coinciden en que es necesario un cambio de actitud y una serie de reformas en los diferentes ámbitos sociales, para que el proceso de atención a la diversidad se dé en forma idónea e integral. Como respuesta a dicha situación, se plantea una propuesta educativa para atender la diversidad en el contexto de aula, por medio de la aplicación de una serie de talleres, que utilizan actividades didácticas participativas, y favorecen el trabajo cooperativo y la construcción propia y colectiva del conocimiento.

La propuesta responde a un enfoque socioconstructivista e inclusivo, pues intenta adaptarse a las demandas de las y los estudiantes y del contexto. Se abordarán los más relevantes ámbitos y aspectos que contempla la diversidad, tomando en cuenta aspectos endógenos y exógenos del individuo. Se espera con esta propuesta que las y los estudiantes manifiestan actitudes de respeto hacia la diversidad, respondan a sus necesidades educativas, las de sus compañeros y compañeras y finalmente, que posean

Máster en Pedagogía con énfasis en Diversidad en los Procesos Educativos del CIDE-UNA. Especialista de Educación Especial. Como docente, ha trabajado para varias universidades y con el Ministerio de Educación Pública. 
una actitud abierta, flexible, crítica hacia sí mismos y su entorno.

Por lo tanto, la formación de las futuras generaciones debe contemplar esta serie de habilidades y destrezas, para que visualicen este mundo como una ventana abierta a la superación individual y colectiva.

\section{Palabras claves}

Diversidad, constructivismo, inclusión, educación, género y equidad.

\section{ABSTRACT}

At the present time, schools face a big challenge: providing their students an education of quality and an education for everyone, in other words, an education thought for diversity within the classroom. The goal of this project is to improve the respect for the students' individual differences and make sure that diversity could be applied in the school context. Based in a diagnostic process developed at "Escuela Mixta San Juan", it was found that most students show rejection and disrespect in all aspects toward others' individual differences.

Authors such as Meléndez (2002), Parcerisa (2000), Olivé (1999) and Aguilar (2000) were revised and they provided some guides to be use at schools in order to deal with the students' diversity. These authors propose that a change of attitude and several different social transformations are required in order to warrant that diversity is appropriately taken care of. As an answer to this situation, a series of workshops and didactic activities designed to improve the cooperative knowledge production and mutual work are proposed.

The proposal is based on a socioconstructivist and inclusive approach, since it attempts to respond to the students' needs within their own context. The most important aspects of diversity were taken into account, such as internal and external aspects related to the individuals. As a final goal, students are expected to express attitudes of respect towards diversity, respond to their classmates and their own educational needs, as well as show an open, critical and flexible attitude towards themselves and their environment.

Therefore, future generation's education must include these skills and abilities, in order to allow them to comprehend this world as an open window to individual and collective improvement.

\section{Key Words}

Diversity, individual differences, school context, proposal, workshops, socioconstructivist, education.

\section{“...TUVE UN SUEÑO..."}

n los últimos años, las diferentes culturas en el mundo, se han visto inmer-
sas en un proceso de acelerados, constantes y significativos cambios. Los cámbios van desde los niveles macro, hasta los niveles micro de un sistema. Es 
un periodo lleno de dicotomías, convergencias y divergencias, de estructuras complejas, de controversias, de políticas globalizadoras, entre otros aspectos propios de esta época. Es aquí donde la diversidad cobra una visión importante dentro de este nuevo mundo, ya que se ve la diferencia como aspecto fundamental en la identidad y la individualidad de cada persona o grupo social. Toda esta panorámica nos abre las puertas hacia un futuro incierto, pues el derecho a las diferencias constituye la base de la cultura actual, donde ya nada es impuesto o preestablecido para un contexto o grupo social dado. Además, da la oportunidad a todas las poblaciones de agrandar sus conocimientos y a mantener un pensamiento crítico, complejo, flexible y dinámico ante los cambios dados.

Martin Luther King, famoso personaje de la historia mundial y defensor de los derechos de los esclavos en los Estados Unidos, en una de sus disertaciones en pro de movimientos emancipadores para la obtención de sus derechos, utilizó la frase: ... “yo tuve un sueño”. Haciendo eco de tal expresión, este trabajo ${ }^{2}$ intenta cumplir el sueño, el cual consiste en conocer, comprender y dar atención a la diversidad en todas sus manifestaciones. Quizá, este sentir sea generalizado en muchos y muchas profesionales en el campo de la educación; pues ha sido y es una utopía para los educadores brindar la atención adecuada a la diversidad de estudiantes que se encuentran en las aulas.

La diversidad y su atención han sido una temática discutida y analizada en los diferentes congresos, cumbres, seminarios en el campo de la educación de los últimos años. Por eso, la atención a las características de las personas en general, vista desde cualquier perspectiva, hoy constituye un factor relevante que debe ser considerado en un proceso de enseñanza y de aprendizaje.

Con la Declaración Universal de Derechos Humanos de las Naciones Unidas en 1948, la Convención de Educación para Todos (Jomtien, 1990) y la Declaración de Salamanca (UNESCO, 1994) empieza a cambiar la visión de las personas que, por una u otra circunstancia, enfrentan situaciones de exclusión en el contexto educativo. Muchos países como respuesta y apoyo a dicha situación, han creado diversas legislaciones con el objetivo principal de ofrecer una mejor calidad de vida en igualdad y equidad de condiciones para todos. Como consecuencia de las directrices adoptadas, se han dado cambios en las diferentes estructuras sociales, laborales, económicas y educativas, logrando

2 Este artículo está basado en un trabajo de graduación realizado en el Centro Educativo Escuela Mixta San Juán, Puriscal, para optar por el grado académico de Maestria en Pedagogia con Énfasis en Atención a la Diversidad de los Procesos Educativos. 
así ampliar el campo de acción a toda esa gama de personas que, por sus características y particularidades, les han sido negados sus derechos fundamentales.

La diversidad comprende una gran variedad de ámbitos y aspectos que influyen en el individuo y en su propia construcción de la identidad. Algunos de los principales ámbitos que la contemplan son: diversidad por estilos y ritmos de aprendizaje, por aspectos personales, por aspectos culturales, sociales, ocupacionales, de género, del entorno demográfico y del entorno ecológico.

Los ámbitos antes mencionados serán analizados, cada uno de ellos con el fin de aportar una visión más real y concreta de cómo interfieren en los procesos de enseñanza y aprendizaje, brindado información importante al docente, la cual puede considerar cuando atiende las necesidades de sus estudiantes. Este documento se convierte en un instrumento o herramienta valiosa, que todo profesional de la educación puede conocer y aplicar en su labor diaria. Esta diversidad supone un reto para el sistema educativo, ya que exige que este adopte un modelo que posibilite el acceso al currículo de todo el estudiantado; esto implica aceptar las diferencias individuales y grupales y ofrecer respuestas educativas ajustadas a sus características y necesidades. Por lo tanto, aceptar la diversidad humana significa brindar a todos los y las estudiantes el mismo tipo de atención en equidad, calidad y oportunidades para participar en la vida escolar, familiar, comunal y social.

En este sentido, la escuela para todos aparece como una respuesta educativa en la lucha contra la exclusión, convirtiéndose el respeto y la igualdad en principios que la fundamentan. De acuerdo con lo anterior, la escuela para todos, al asumir el derecho a la educación en su sentido más pleno e integrador, pretende promover un cambio no sólo en el currículo y la enseñanza, sino también en la organización de los centros y del propio sistema educativo. Además, que la y el estudiante conozcan y comprendan el concepto de diversidad, aplicado a sí mismo y al de sus compañeros y compañeras.

Por todo esto, se pretende sensibilizar a la población estudiantil con respecto al tema de la diversidad y sus ámbitos como elemento integrante e inherente al ser humano; se parte de la idea de que las personas son diversas y, por lo tanto, deben ser respetadas, de acuerdo con sus características, su forma de vida, su cultura, sus aspectos sociales, demográficos y ecológicos.

\section{¿Por qué se plantea la propuesta en el ámbito del aula escolar?}

El trabajo podría implementarse en cualquier centro educativo, por medio de un proyecto educativo didáctico que sensibilice, tanto a las y los 
docentes, como a las y los estudiantes, sobre el concepto de diversidad y todo lo que implica conocerla, comprenderla y vivenciarla. La propuesta se plantea en el contexto del aula, ya que la formación de las futuras generaciones en el campo de la diversidad, aseguraría personas con mentes abiertas al cambio, con un mayor nivel de respeto, tolerancia y flexibilidad para interactuar.

Es importante mencionar que esta población se encuentra en una etapa de conformación de su identidad y de construcción de su entorno, lo que permitiría una mayor accesibilidad hacia el reconocimiento del otro, como sujeto. También, es en el contexto de las aulas propiamente, donde se ubican estudiantes con una gran variedad de particularidades y necesidades educativas derivadas de aspectos sociales, individuales y culturales principalmente. No obstante, resulta necesario y pertinente que el personal administrativo y docente se haya capacitado con anterioridad en el campo de la atención a la diversidad, con el fin de que cumplan la función de apoyo para dicha intervención. Muchas de estas capacitaciones, ya se han introducido en forma empírica y paulatinamente, con ideas tendientes a ver las características de cada persona, así como las de grupo como un aspecto más dentro del marco de la atención a la diversidad, las cuales pueden encontrarse en una clase o una comunidad educativa.

La propuesta se orienta al trabajo con estudiantes de primaria, por ser el primer escalón donde debe abordarse el tema del respeto a las diferencias individuales desde una perspectiva de la diversidad. Se considera pertinente dar un seguimiento a la población y al centro educativo que ponga en funcionamiento este plan piloto y, cuando esté cubierta toda la población estudiantil, se incluyan nuevas etapas de trabajo, para el abordaje del tema de la diversidad, su comprensión y su vivencia de forma paralela y conjunta con el personal docente y administrativo de la institución y la comunidad.

Esta última etapa de trabajo surge como respuesta a una investigación realizada por el Instituto de Investigaciones Psicológicas de la Universidad de Costa Rica, en el año 2003, que aunque no hace referencia específica de la temática de la diversidad en general, sí considera uno de los aspectos en este ámbito.

En esa investigación, se evidenció que existen sentimientos de rechazo, temor e inseguridad en las y los docentes y administradores para atender a estudiantes con necesidades educativas especiales dentro del sistema regular. Este sentir ha tomado auge y se ha generalizado en la mayoría de los centros educativos, por ende, la necesidad de crear un plan didáctico de trabajo para abordar los diferentes aspectos que contemplan la diversidad. 
Se eligió para ello un centro educativo rural, con una cantidad importante de estudiantes, en el cual estuviera presente la mayoría de aspectos y ámbitos que comprenden la diversidad. Este trabajo inició con un proceso de diagnóstico e investigación de las características de infraestructura, geográficas, demográficas, culturales del centro educativo, así como de las particularidades generales de la comunidad estudiantil y comunal.

\section{¿Qué se identificó como situación problema?}

Los y las estudiantes del centro educativo donde se llevó a cabo el proceso de investigación, conceptúan la diferencia como una enfermedad, una situación inherente a la persona misma y donde la sociedad no tiene injerencia en dicha situación. La anterior percepción lleva a una serie de actitudes discriminatorias por parte del estudiantado con respecto a las diferencias individuales, desde el ámbito escolar, comunal, laboral y social. Por tal motivo, se ha decidido plantear una propuesta que pretenda sensibilizar al estudiantado con respecto al reconocimiento de sus propias diferencias y las de sus compañeros y compañeras, desde una perspectiva de la diversidad y que estas diferencias dejen de ser vistas sólo por el género, la nacionalidad, la religión o condición discapacitante, como tradicionalmente se ha enfocado.

Lo anterior fue resultado de un diagnóstico realizado, cuyo objetivo era conocer la opinión que tienen los estudiantes con respecto a las diferencias individuales dentro del marco de la diversidad. La muestra seleccionada tenía como fin conocer la visión general de esa comunidad estudiantil. Considerando los resultados obtenidos en dicho proceso diagnóstico, se concluye lo siguiente:

Las y los estudiantes, en general, se encuentran sensibilizados con respecto a las diferencias individuales, pues manifiestan actitudes positivas hacia estas; lo que se plasma principalmente en las respuestas de estudiantes del segundo ciclo. Quienes cursan el primer ciclo reconocen las diferencias como algo propio del individuo, catalogadas como buenas o malas.

La población identifica la diferencia referente a lo concreto y tangible, por lo que es necesario ahondar sobre la diversidad y los aspectos que la conforman, con el objetivo de que los alumnos y las alumnas reconozcan y vean la diferencia presente en todos y en todo. Es decir, forma parte de la naturaleza humana.

El aspecto de género y sexo debe ser abordado en casi todos los niveles, pues para ambas concepciones se manejan pensamientos estereotipados, los 
cuales reflejan tendencias discriminatorias hacia la igualdad entre los hombres y las mujeres. Lo anterior como consecuencia de los contextos socioculturales que vivencian las y los estudiantes y la sociedad en general.

En síntesis, a partir de los resultados encontrados en la identificación de la situación problema, se considera, para efectos del planteamiento de la propuesta, tomar en cuenta lo siguiente:

Resulta necesario realizar trabajos con los niveles inferiores del centro educativo, sobre el concepto de diversidad y sus implicaciones en los diferentes contextos, para que conozcan y adquieran un conocimiento de lo que implica el respeto a la diversidad. Podría plantearse el tema de la diversidad como eje transversal presente en la vida de todo ser humano.

Incorporación en los planeamientos de temas como diferencias individuales, diversidad, valores y necesidades educativas.

Provisión a las y los estudiantes de estrategias y apoyos para interactuar de la manera idónea con cualquiera de sus iguales, sin importar sus capacidades y que den respuesta a sus necesidades educativas, de acuerdo con las posibilidades del contexto en que se desenvuelven.

La educación actual enfrenta grandes dificultades en cuanto a su eficacia, calidad y eficiencia. Hoy, los niveles de deserción escolar en primaria y secundaria son muy altos, lo que preocupa a todos los profesionales que laboran en el área. Dentro de las investigaciones realizadas en el marco de esta problemática, no sólo en el ámbito nacional, sino que también en el internacional, se ha determinado que una de las grandes deficiencias que enfrentan los centros educativos, radica en el desinterés por conocer, comprender y aceptar las características individuales y colectivas que poseen las personas. En otras palabras, existe una gran problemática en los diferentes centros educativos por responder a las necesidades educativas que los y las estudiantes presentan durante el proceso de formación académica.

Es importante mencionar que muchos de los apoyos que las y los estudiantes presentan son derivados de causas externas, como por ejemplo, de las condiciones que ofrece el medio para desenvolverse, aspecto que la mayoría de veces se deja de lado y todo recae sobre el individuo mismo. Aunado a esto, estudios revelan que el respeto a la diversidad en su sentido más amplio no se vivencia, ya que algunas personas enfrentan a diario serias limitaciones a causa de su condición personal, social o cultural. De ahí que no se reconoce la diversidad como parte inherente al ser humano y al medio en que se desenvuelve.

Otro factor que influye se debe a que los y las docentes o encargados de los procesos de formación de centros educativos, no atienden las necesidades 
que presentan cada uno de sus estudiantes dentro de las aulas, ya sea por falta de recursos, recargo de estudiantes en el aula o por desinterés, lo que viene a empeorar la calidad de la educación.

Con un paradigma sociohistórico, modelo inclusivo y un enfoque constructivista, la comprensión y la vivencia de la diversidad debe basarse en el respeto, la equidad, la igualdad, la tolerancia y la participación, las cuales constituirían sus pilares y responderían a una educación para todos; es decir, a la educación en valores, en especial, los valores de la diversidad.

Aguilar (2000, p. 90) menciona: "El Estado tiene la obligación de respetar los derechos de grupo y hacer que se les respete en virtud de una cultura auténtica y autónoma, pero, al mismo tiempo, el Estado tiene la obligación con los ciudadanos de reconocer, respetar cada uno de los derechos individuales". Y son precisamente derechos como la igualdad, la equidad, el respeto, la tolerancia, entre otros, los que potenciarían una sociedad inclusiva e independiente.

En otras palabras, comprender la diversidad implica el reconocimiento y el respeto de una cultura, de cada uno de sus miembros, donde estos puedan vivir según lo que cada quien elija, de acuerdo con las características propias individuales y de su contexto. Por ende, la diversidad orienta la educación hacia los valores de igualdad, justicia, respeto, libertad, estableciendo así un compromiso por parte de todos para con los grupos y culturas "minoritarios".

\section{Una concepción de diversidad}

La diversidad responde a la idea de que todos los seres humanos son diferentes y dichas diferencias, lejos de imponer grados de jerarquía, se constituyen como elementos enriquecedores del mundo, es decir, donde todos tienen algo que aportar al medio. La comprensión y el respeto de esta, implica toda una transformación en los diferentes ámbitos de la sociedad. Cabe mencionar que gracias al surgimiento de algunos movimientos emancipadores, muchos grupos subordinados han mejorado sus condiciones de vida, en una sociedad llena de desigualdades.

Meléndez (2002) define la diversidad humana como:

Condición de la vida en comunidad, cuyos procesos vitales se relacionan entre sí y se desarrollan en función de los factores culturales de un mismo ambiente. Donde, tanto relación como variedad, aseguran y potencian la vida de todos los habitantes de esa comunidad (p. 28). 
Según Meléndez (2000, p. 34), comprender la diversidad, no sólo se refiere al respeto y a la aceptación de las diferencias individuales, sino que debe darse una serie de cambios en todas las estructuras, sociales, políticas, económicas y educativas de un sistema, para que la potencien. Es decir, una asociación de niveles distintos.

Este autor hace una clasificación de los aspectos de la diversidad; sin embargo, no son los únicos, pues abordar la diversidad tomando en cuenta todos sus ámbitos resulta una tarea interminable. En este documento, se intenta considerar los más relevantes.

Diversidad de género: contempla y valora el ser humano en lo referente a su sexo, condición de género y su preferencia sexual.

Diversidad de estilos de aprendizaje: los estilos de aprendizaje, se refieren a la forma particular en que aprenden los individuos y cómo procesan la información.

Diversidad de niveles de conocimiento: toma en cuenta el nivel de conocimiento de partida o el estadio en que se encuentra el individuo para la enseñanza. Este aspecto va relacionado con el nivel de desarrollo humano del estudiante, su proceso madurativo, los conocimientos previos, destrezas y habilidades.

Diversidad de ritmos de aprendizaje: referido al tiempo que tarda el o la estudiante para realizar las tareas asignadas y el tiempo en que logra codificar la información que recibe.

Diversidad personal individual: en este aspecto, se consideran todas las características personales de cada quien, como un ser único e irrepetible, en especial sus condiciones biológicas. Algunas de las variables utilizadas para valorar este aspecto son: la edad, las necesidades educativas de cada persona o cualquier situación particular del individuo.

Diversidad cultural: aquí se ubican los aspectos referidos a las relaciones culturales y multiétnicas del individuo. Algunas variables que podrían considerarse son las siguientes: nacionalidad, domicilio, credo religioso, costumbres, idioma y tradiciones.

Diversidad social: dentro de este aspecto, se ubican las siguientes variables: la familia, también grupos formales y no formales presentes en la comunidad y demás organizaciones humanas en las que participan las personas de su medio.

Diversidad ocupacional: son las fuentes de enriquecimiento y supervivencia de los individuos, los oficios a los que se dedica el niño y la niña, sus progenitores en su contexto y el nivel socioeconómico del núcleo familiar. 
Diversidad y entorno demográfico: en este aspecto se anota una serie de características que prevalecen en la comunidad, la composición poblacional autóctona y el punto de encuentro con demás grupos que la complementan.

Diversidad y entorno ecológico: este aspecto contempla los recursos naturales con los que el individuo interactúa, los servicios brindados por el centro educativo a las y los estudiantes, así como el reconocimiento y respeto por la biodiversidad.

Al respecto, Sacristán citado por Parcerisa (2000) considera que:

La diversidad alude a la circunstancias de los sujetos de ser distintos $y$ diferentes (algo que en una sociedad tolerante, liberal y democrática es digno de ser respetado). Aunque también hace alusión, por otra parte, a que la diferencia sea, en realidad, desigualdad, en la medida en que las singularidades de los sujetos o de grupos les permitan a éstos alcanzar determinados objetivos en las escuelas y fuera de ellas en desigual medida (p. 12).

Este autor analiza las diferentes perspectivas por las que la sociedad puede ver la diversidad; hace notar cómo, a pesar de las condiciones de desventaja en que viven los individuos con respecto a la norma, esta población es sumamente capaz de aportar y dar, de acuerdo con las posibilidades de su contexto.

\section{Educación y diversidad}

La educación tiene como finalidad promover el desarrollo de capacidades y la adquisición de contenidos necesarios para que el individuo logre desenvolverse, de la mejor manera, en los diferentes ámbitos. Por ende, la escuela debe mantener un equilibrio entre el contexto, la cultura y las características propias del individuo, con el fin de que ofrezca una respuesta educativa acorde con las necesidades de cada estudiante. Dicha oferta debe ser comprensiva y diversificada, que evite la discriminación, la desigualdad de oportunidades y respete las características y las necesidades individuales.

Es importante considerar que en el proceso educativo, las necesidades educativas de los y las estudiantes son de carácter interactivo, lo que implica que son cambiantes, porque pueden variar en función de la evolución de los propios educandos o de la respuesta educativa que se les ofrece. La escuela tiene, por lo tanto, un papel fundamental para evitar que las diferencias individuales constituyan un elemento discriminatorio y generadoras de desigualdades educativas. 
Sin embargo, resulta necesario aclarar que corresponde tanto al sistema educativo como a otros entes institucionales, realizar reformas en sus estructuras, para que realmente exista una verdadera equidad. Se requiere una coordinación entre todas las estructuras, con el fin de que la población en condiciones desiguales acceda a los diferentes entornos en equidad de condición.

La escuela de la diversidad se centra en saber aprender estrategias para la resolución de problemas de la vida cotidiana, de manera cooperativa y solidaria. Al respecto, Meléndez (2000, p. 24) menciona que "educar y educarse en condiciones de diversidad solidaria, posibilita procesos de conocimiento y consolida los principios de equidad y de no discriminación para un desarrollo global...". El desafío consiste en brindar condiciones de una escuela para todos; todos los niños, jóvenes y adultos tienen derecho a la educación y no son nuestros sistemas educativos los que tienen derecho a seleccionar ciertos tipos de niños; es el sistema el que debe ajustarse para satisfacer las necesidades de todos.

La comprensión de la diversidad, en la mayoría de los centros educativos, aún no empieza, por lo que es necesario concientizar a todos los involucrados dentro del proceso, sobre el respeto de las diferencias individuales. Todo centro posee una infinidad de características y condiciones que están presentes en la vida de las y los estudiantes, las cuales influyen directamente en cada una de las actividades que realizan. Por ende, es necesario que se conozcan aquellos aspectos comprendidos en la diversidad escolar, para mejorar la calidad de la educación que reciben los educandos.

\section{Una escuela en y para la diversidad}

Coronel (2001, p. 5) manifiesta que "una educación para la diversidad es un programa ambiguo con aplicaciones muy distintas: diferentes métodos para sujetos desiguales, escuelas diversas para sujetos encajados en culturas varias: currículos desemejantes para escuelas, sujetos y culturas distintos". La escuela debe constituir un lugar de ruptura y de apertura con el medio de origen de las y los escolares; el educador tendrá una función mediadora entre los educandos, el medio y los valores universales. Por lo tanto, es importante considerar las experiencias y el bagaje cultural que posee cada estudiante.

Aguilar (2000, p. 116) enfatiza que para atender la diversidad escolar es necesario llevar a cabo un conjunto de intervenciones educativas, que desde una oferta educativa básicamente común para todos las y los estudiantes, ofrezca respuestas diferenciadas para cada uno y ajustadas a sus características individuales y de su contexto. 
Dicho autor plantea diez requisitos necesarios, como herramientas básicas a contemplar dentro del proceso de reforma educativa. Estos son los siguientes:

- $\quad$ Entender la noción de diversidad en su sentido más amplio.

- Partir de una evaluación inicial que permita conocer el nivel de competencia curricular del estudiante.

- Tomar en cuenta los cinco tipos de capacidades (cognitiva, afectiva, psicomotriz, motivacional, la relacional y de inserción).

- Aceptar la peculiaridad de los y las estudiantes.

- Asumir la idea de que no existen especialistas para atender la diversidad y que es responsabilidad del docente dar respuesta a esta.

- Adoptar una metodología diversificada.

- Utilizar y conocer los diferentes apoyos que podrían dar respuesta a las necesidades educativas presentes en el estudiantado.

- Incorporar las directrices y medidas para la atención de la diversidad dentro del contexto interno del centro educativo.

- Una respuesta educativa para la diversidad no implica sólo cambios en los apoyos; va orientada hacia un cambio de paradigma y una perspectiva más equitativa y justa. Es necesario deshacerse de los juicios previos y partir de actitudes positivas ante las diferencias.

De acuerdo con lo anterior, Aguilar (2000, p. 170) sugiere para la atención de la diversidad en el aula, los siguientes aspectos, relacionados con:

- Los objetivos: deben ser explícitos y formularse en términos de capacidades.

- Los contenidos: contemplar los tipos de contenidos (conceptos, procedimientos y actitudes), dar más atención a los contenidos que representan mayor grado de dificultad para el o la estudiante y fortalecer el aprendizaje significativo y el uso de unidades didácticas integradas.

- La metodología, la organización y los recursos: se anotan la combinación de distintos modos de agrupamiento, el uso de estrategias metodológicas diversas y el uso de variedad de recursos.

- Las actividades: consideran el horario para desarrollar las actividades, su planteamiento durante la clase, el uso de actividades de refuerzo y que despierten interés, la motivación y el autoconcepto positivo. También, actividades que admitan la investigación, faciliten los procesos 
intelectuales y fomenten el planteamiento de actividades con diferentes grados de dificultad.

- La evaluación: una evaluación continua, donde las estrategias, actividades e instrumentos sean variados. Los criterios de promoción y titulación deberán dejar un margen abierto al debate y a la evaluación cualitativa por parte del docente.

Aguilar cita una serie de lineamientos con respecto a cómo deben llevarse a cabo los procesos de enseñanza y de aprendizaje en un contexto inclusivo, donde valora y enfatiza, principalmente, el papel de la y el estudiante como actores principales y cada docente como mediador y encargado de potenciar aprendizaje.

Otros aspectos importantes que ha de poseer una escuela para atender y respetar la diversidad, según Vega (2001, p. 52), son los siguientes:

- La escuela para todos:

- Debe ser más abierta a la comunidad.

- Debe replantear su currículum para que responda a las diferentes necesidades.

- Implica la participación activa de los padres y la cooperación con otras instituciones y servicios comunales.

- Integra en sus proyectos nuevas redes y servicios de apoyos, de acuerdo con las necesidades de todos los miembros de la comunidad.

- Considera la flexibilidad como recurso para la optimización del proceso educativo.

\section{Modelo pedagógico}

Para que un centro educativo comprenda, reconozca y atienda la diversidad, es importante que se consideren los siguientes aspectos:

La aceptación de las diferencias individuales entre los niños y las niñas con respecto a la cultura, el estilo de aprendizaje, los intereses, las necesidades específicas y los valores de cada persona.

La cooperación es indispensable en los procesos de atención a la diversidad, pues desarrolla en las y los estudiantes importantes habilidades sociales y cognitivas que se adquieren en un contexto educativo que atienda a la diversidad. 
La criticidad en cada estudiante y su capacidad de comprender que todos tenemos habilidades y necesidades específicas que enriquecen un contexto escolar, cultural y social.

La diferencia no sólo debe ser vista desde el aspecto de la discapacidad. La diferencia abarca todo lo que somos, nuestra forma de pensar, de actuar y de ser.

El contexto histórico de todo el alumnado es importante, para poder dar una atención acorde con las experiencias vividas.

La atención de las necesidades educativas, para desarrollar las capacidades y habilidades básicas de los alumnos, respeta sus características personales y referencias socioafectivas.

La pluriculturalidad que se vive en las escuelas es un elemento enriquecedor para la sociedad, ya que da oportunidad a las personas de compartir sus experiencias y de participar activamente en otras sociedades.

Entonces, un ejemplo de modelo pedagógico que sea compatible con una escuela en y para la diversidad, debe tomar en cuenta lo siguiente:

- Enfoque socioconstructivista.

- $\quad$ Modelo multicultural.

- Currículo alternativo y flexible.

- Trabajo cooperativo.

- Docente mediador.

- Aprendizaje significativo.

- Aprendizaje recíproco.

- Respeto de las diferencias individuales.

- $\quad$ Educación como un proceso dinámico y participativo.

Por todo lo anterior, se determina que los procesos de enseñanza y aprendizaje constituyen un elemento trascendental dentro del proceso de formación de los y las estudiantes, pues se busca el desarrollo integral del ser humano y con ello el de la sociedad. Estos se orientan al hombre y a la mujer, quienes necesitan adquirir y transmitir su cultura, definir sus valores, formar un concepto claro del mundo en que viven y desarrollar sus potencialidades para vivir una vida plena.

Recordemos que "el problema" no es la diversidad, si no los medios, los recursos, la infraestructura, los aspectos ideológicos y los culturales provocan que las personas en situación desventajosa enfrenten diversas barreras, impuestas por la sociedad. Sin embargo, no todo es negativo; se empieza a 
notar que ciertos centros educativos promueven una escuela para todos y tratan de adaptar el medio a las características de la comunidad estudiantil. Además, por medio de la vivencia de valores en la escuela, comunidad y familia, se forma a las futuras generaciones sobre el respeto a las diferencias individuales, la comprensión y vivencia de la diversidad.

\section{Un cambio de actitud hacia la diversidad}

A lo largo de la historia, las personas con características significativamente diferentes con respecto a las de sus semejantes, consideradas por muchos como "anormales", han enfrentado continuas situaciones y condiciones de desventaja ante los demás. Algunos organismos ante dicha situación han tratado de atender la diferencia, pero esto no ha sido suficiente. Es necesario tener presente que no basta con brindar los diferentes tipos de apoyos o de reemplazar modelos de atención, sino que se requiere de un verdadero compromiso por parte de toda la población, para que se promuevan el reconocimiento y la vivencia en equidad e igualdad de condiciones para cada persona.

A modo de ejemplo y continuando con el tema de la atención de la población con discapacidad, es pertinente acotar que, aunque teóricamente, se dice que si se brinda una oportuna atención a las necesidades educativas derivadas de una condición discapacitante, estas ya no estarían presentes. La realidad refleja que no es así. En muchos estudiantes con discapacidad, a pesar de los apoyos que reciben en los centros educativos, sus necesidades educativas aún prevalecen, lo que denota que estas medidas no son suficientes.

También, los organismos de carácter internacional se han encargado de legitimar concepciones erradas con respecto a este tema. Por ejemplo, la definición de discapacidad que ha sido utilizada durante las últimas décadas, por la Clasificación Internacional de Deficiencias, Discapacidades y Minusvalías (CIDDM, 1980), hace referencia a este término como: "toda restricción o ausencia de la capacidad de realizar una actividad dentro de los parámetros considerados como normales, debido a una deficiencia". La Ley 7600, emitida en 1996, la define así: "cualquier deficiencia física, mental o sensorial que limite, sustancialmente una o más de las actividades principales de un individuo" (p. 8). En ambos casos, se denota como la condición recae sobre la persona, como si esta fuese la única causante de la discapacidad y deja de lado aspectos ambientales que podrían generar una condición discapacitante.

Hoy, bajo el paradigma inclusivo y dentro del marco de la atención a la diversidad, se ve la discapacidad como una condición que no sólo enfoca 
al individuo que la presenta, si no que involucran las características que el medio le ofrece para desenvolverse. Lo anterior lo ratifica la Clasificación Internacional del Funcionamiento, de la Discapacidad y de la Salud de la Organización Mundial de la Salud (CIF, 2001), la cual define discapacidad como aquella que:

Indica los aspectos negativos de la interacción entre un individuo con una condición de salud dada y los factores contextuales (ambientales y personales). Es un término genérico que incluye deficiencias en las funciones y estructuras corporales, limitaciones en las actividades y restricciones en la participación. Al igual que el funcionamiento, se entiende como una interacción dinámica entre la interacción de salud y los factores contextuales.

Esta definición sobre discapacidad considera los aspectos propios de la deficiencia causantes de la discapacidad y contempla la repercusión del entorno social y físico en el funcionamiento del individuo. Esta definición perfectamente responde a un modelo universal, integrador, interactivo e inclusivo.

Se ha notado que la población con discapacidad se ha visto rechazada y discriminada a lo largo de la historia por motivo de las diferencias que presentan respecto a la "norma"; sin embargo, gracias a los procesos de integración escolar es que ha cambiado, de manera paulatina, esa visión negativa que se poseía. Ante este panorama, un cambio de actitud, en primera instancia, por parte de las personas, es lo que constituye los cimientos para promover y proponer una escuela en y para la diversidad.

Con este trabajo investigativo, se plantea la transformación de una escuela selectiva a una escuela inclusiva; la primera se concibe como ente reproductor de las desigualdades sociales, pues se encuentra atada por reglamentos inflexibles, cuyas normas de promoción se basan en la normalidad y normatividad. La segunda es aquella que ofrece alternativas educacionales a las y los estudiantes, por medio de un currículo que se acerque a las posibilidades y necesidades de estos, citado por Meléndez (2002).

Por lo tanto, analizando debilidades y potencialidades de la comunidad institucional y ante el reto que planteaba el sueño de atender la diversidad, se emprende un nuevo camino hacia una escuela para todos, con una propuesta educativa que aborde la diversidad en el aula. En seguida se presenta un extracto de esta. 


\section{Una propuesta educativa para abordar la diversidad en los contextos de aula}

La propuesta educativa que busque abordar el tema de la diversidad en el ámbito curricular y de aula, debe comprender y vivenciar el respeto y la atención a la diversidad. Esta ha de orientarse al abordaje del tema de la diversidad y los aspectos que la constituyen, con el fin de que las y los estudiantes sean capaces de comprender que las diferencias se manifiestan en todo los ámbitos en que el ser humano interactúa y que, por lo tanto, es una condición inherente en cada persona.

Tendrá el propósito de implementar, dentro del Centro Educativo, una serie de actividades con el fin de conocer el concepto de diversidad con el estudiantado, para lograr que este posea una visión crítica sobre lo referente a las diferencias individuales, así como un cambio de actitudes en relación con el trato hacia la otredad, utilizando valores como respeto, igualdad y equidad.

\section{Descripción general de la propuesta}

La propuesta está constituida en un plan de trabajo grupal de nueve semanas como mínimo, divida en igual cantidad de talleres. En dichos talleres, se aborda el tema de la diversidad, su comprensión y su vivencia, considerando los diferentes contextos en que los y las estudiantes se desenvuelven.

Los objetivos principales que la constituyen son los siguientes:

- Generar cambios de actitudes en los y las estudiantes en relación con el trato hacia las diferencias individuales, dentro de! marco de la diversidad y de los valores del respeto, la igualdad, la tolerancia y la equidad, en los diversos contextos.

- Concientizar a las y los estudiantes sobre el respeto de las diferencias individuales como parte de la diversidad humana.

- Proveer a las y los estudiantes de aiternativas didácticas a considerar en diferentes contextos, que fomenten el respeto, la igualdad y equidad hacia la diversidad escolar.

- Brindar a las y los estudiantes alternativas didácticas que respondan a sus propias necesidades educativas y las de sus compañeros de grupo, las cuales sean funcionales en los diversos entornos. 
Los talleres de mediación pedagógica utilizan actividades como reflexiones, entrevistas, juegos y dinámicas dentro de clase, las cuales permitan una participación activa durante del proceso. Se concibe el taller como tiempo y espacio para la vivencia, la reflexión y la retroalimentación de todos sus participantes, y el lugar para la participación y el aprendizaje. El taller, en síntesis, puede convertirse en el lugar del vínculo, la participación, la comunicación y, por ende, lugar de producción social de objetos, hechos y conocimientos.

Esta definición respalda y fomenta la atención de la diversidad en los contextos educativos, pues permite la valoración de cada participante tomando en cuenta su individualidad y promoviendo la construcción de conocimientos a partir de la interacción con los demás.

Los contenidos que trabaja la propuesta, orientada a la atención a la diversidad, son los siguientes:

- Concepto de diversidad, considera el conjunto de características que cada persona o grupo social posee, haciéndolo irrepetible y las cuales son importantes para el mejoramiento de una sociedad, donde todos, sin importar sus capacidades, pueden aportar para el beneficio de una comunidad.

- Los ámbitos de la diversidad en general, se subdividen en:

- Ámbito personal o individual de la diversidad.

- Estilos de aprendizaje: referido a la manera en que cada individuo aprende y lleva la información al cerebro.

- Ritmos de aprendizaje: tiempo en que tarda el o la estudiante para realizar las tareas asignadas.

- Nivel de conocimiento: conocimiento inicial en que se encuentra el o la estudiante para la enseñanza.

- Género: se valora al ser humano en lo referente al sexo, su preferencia sexual y rol social.

- Aspectos personales: todas las características personales de la persona.

- Ámbito sociocultural de la diversidad.

- Entorno ecológico: se refiere al contexto natural en que se desenvuelve el individuo y los servicios que accede.

- Entorno demográfico: toma en cuenta las características de la comunidad, composición de la población.

- $\quad$ Aspecto social: se manifiesta por medio de la organización social que prevalece en la comunidad y en la que el individuo participa. 
- Aspecto cultural: se refiere a las costumbres, tradiciones, formas de vida de los miembros de una comunidad.

- Valores que contempla la diversidad:

- Respeto: valoración de todas las personas sin importar sus condiciones sociales, familiares.

- Igualdad: acceso que debe tener toda persona a los servicios de la comunidad, sin ningún tipo de discriminación por determinada circunstancia.

- $\quad$ Equidad: se refiere a la disposición de servicios de forma proporcional a todo individuo.

- Justicia: brindar al individuo lo que merece según su situación y sus posibilidades.

- Tolerancia: se refiere a la aceptación y valoración de lo que caracteriza a otro individuo y que, por lo tanto, lo hace particular.

- Necesidad educativa concebida como: las dificultades o barreras que presentan algunas personas o su entorno, para acceder a los diferentes servicios con los que cuenta la comunidad. Puede ser originada por aspectos personales o ambientales.

- Con respecto a las estrategias pedagógicas, se utilizan las siguientes:

- Trabajo cooperativo: es trabajar en un pequeño grupo, compartiendo responsabilidades, intereses, habilidades, objetivos.

- Compañero tutor: referido al apoyo constante y mantenido de un compañero durante el período de clase del o la estudiante.

- Discusión en grupo: es una conversación planeada entre tres o más personas, sobre un tema en especial y bajo una dirección.

- Dramatización: es la dramatización, sin ensayo, de un conflicto realizado por dos o más personas para que ese conflicto sea analizado por el grupo.

- Entrevista: una persona es interrogada por un miembro del grupo, sobre un tema que se ha definido con anticipación.

- Lectura comentada: consiste en dejar a los y las participantes leer un documento y que lo comenten con la dirección del instructor.

\section{Instrumental evaluativo para medir los alcances de la propuesta}

La evaluación de dicho proceso responde a criterios procesales, formativos y actitudinales. Se utiliza una bitácora para retomar las acciones sucedidas durante el proceso del taller y una sistematización, donde se 
analiza a profundidad cada uno de los talleres de manera exhaustiva. Además, los y las estudiantes participantes evalúan cada uno de los talleres, por medio de instrumentos elaborados para tal fin. La evaluación final se realiza mediante el uso del mural escolar en el que se proyectan los alcances que obtuvo con la propuesta.

Se espera que después de participar en los talleres involucrados en esta propuesta, los y las estudiantes:

- Manifiesten actitudes de respecto, igualdad y equidad hacia sus compañeros en los diferentes contextos escolares

- $\quad$ Posean un concepto claro de diversidad.

- Identifiquen las necesidades educativas propias y las de sus compañeros y compañeras de grupo.

- Brinden alternativas de apoyo a las necesidades educativas de sus compañeros y compañeras de grupo.

- Mejoren las relaciones sociales entre los miembros de la comunidad educativa.

- Mantengan una actitud positiva ante su proceso de aprendizaje.

- Mejoren su rendimiento académico.

En suma, para que se comprenda y vivencie la diversidad, se requiere que el sistema educativo tome en consideración reformas en su estructura, organización y, primordialmente, en su propuesta curricular, pues exige una transformación social-ideológica donde se dé importancia a las diferencias humanas y estas se vean como elemento enriquecedor de una cultura; el respeto a la diferencia como reconocimiento de ser diverso, es el principio de la transformación social.

Es importante que se ejecuten diversos mecanismos de promulgación institucional y comunal, sobre información referida a diversidad, con el fin de que las personas vayan familiarizándose con nuevos procesos de cambios.

La pluriculturalidad que se vive en las escuelas es un elemento enriquecedor para la sociedad, ya que da oportunidad a las personas de compartir sus experiencias y de participar activamente en otras sociedades.

La educación y la familia, ambas instituciones, conforman la alianza idónea para que la persona logre sus sueños. No perdamos de vista que ambas constituyen las primeras escuelas donde el ser humano adquiere sus primeros aprendizajes. Las familias juegan un papel trascendental en este proceso, ya que nos dan la pausa e incluso podrían funcionar como estrategia 
didáctica y de apoyo, para crear conciencia en el estudiantado, sobre las diferencias entre los unos y los otros; claro, siempre respetando la individualidad de cada uno y fomentando procesos inclusivos, ya no sólo en el nivel escolar, sino también en otros contextos.

Además, es evidente que no es por medio de la creación de nuevas legislaciones, cambios conceptuales, que se van a dar los procesos de cambios necesarios para la búsqueda de atención de la diversidad. Las leyes en este caso, constituyen herramientas o agentes reproductores de un discurso, las cuales en muchos aspectos llevan a la reproducción y en otros, al efecto contrario del fin por el que fueron creadas. Es así como se lleva a la práctica algo que no tiene significado para el ejecutor y, por ello, se fracasa en el intento, e incluso se afecta a un individuo por ejecutar acciones de manera mediocre.

Considerando que el objetivo implícito de la escuela es la adaptación social de los y las estudiantes, el impacto de esta proporciona la base para interactuar con el mundo circundante. Dadas las innumerables combinaciones y amalgamas de experiencias, que se presentan en los procesos de enseñanza y aprendizaje, la escuela llega a tener un significado individual, particular o colectivo, según sea la necesidad que cada individuo manifieste. Desde el punto de vista de la escuela formal, se considera como la instrucción o el proceso de mediación sistemático que niños, jóvenes y adultos reciben como preparación para enfrentar los retos de la vida.

Entonces, se concibe la escuela como un ente encargado de formar personas que puedan incorporarse y desarrollarse en un ambiente o varios ambientes determinados, que contribuye con la autodeterminación de una personalidad, de una identidad cultural y por qué no decir, de una nación.

Es necesario tener presente que esto apenas comienza, que queda mucho por hacer en este campo y que la verdadera fuerza se encuentra en cada uno, en nuestros trabajos, actos, formas de vida, pensamiento, actitudes. Está en manos de los docentes como mediadores de los procesos de enseñanza y aprendizaje, formar a las futuras generaciones con toda esta gama de habilidades y potencialidades, para que puedan ver este mundo como una ventana abierta a la superación personal y no como una amenaza.

\section{"Promovamos un cambio de actitud para luego alcanzar una verdadera aptitud".}




\section{REFERENCIAS}

Aguilar, L. (2000). De la integración a la inclusividad. Buenos Aires, Argentina: Editorial Espacio.

Asamblea Legislativa. (1996). Ley 7600. Igualdad de oportunidades para las personas con discapacidad en Costa Rica. San José, Costa Rica: Consejo Nacional de Rehabilitación y Educación Especial.

Cerdas, A. y Rodríguez, A. (2003). Propuesta de actividades para el desarrollo de las funciones de los docentes de apoyo en retraso mental. Manuscrito no publicado: San José, Costa Rica.

Devalle, A. y Vega, V. (1999). Una escuela en y para la diversidad. El entramado de la diversidad. Argentina: Aique.

Gutiérrez, E. (2004). Aplicación de la terminología propuesta por la Clasificación Internacional del Funcionamiento, de la Discapacidad y de la Salud (CIF). Fundación SIDAR. Retrieved November 14, 2004. From: http:/wwwsidar. org/recur/desdi/pau/cif.php\#def

Instituto de Investigaciones Psicológicas. (2003). Actitudes y creencias de maestros y administradores de preescolar, primaria y secundaria en relación con la atención de las necesidades educativas especiales. UCR. San José, Costa Rica.

Instituto Costarricense de Enseñanza Radiofónica. (1994). Algunas sugerencias para el trabajo en grupos. San José, Costa Rica: Editorial ICER.

Maya, A. (1996). El Taller Educativo. ¿Qué es?, Fundamentos, cómo organizarlo y dirigirlo, cómo evaluarlo. Bogotá, Colombia.

Meléndez, L. (2002). La inclusión escolar del alumno con discapacidad intelectual. Bogotá, Colombia: GLARP-IIPD.

Monereo, C. (2000). Instantáneas. Proyectos para atender la diversidad educativa. Madrid, España: Editorial CELESTE EDICIONES.

Naciones Unidas. (1948). Declaración Universal de Derechos Humanos. Naciones Unidas.

Parcerisa, A. (2000). Atención a la diversidad: claves para la innovación educativa. Barcelona, España: Editorial Graó.

UNESCO. (1990). Conferencia Mundial de Educación para Todos. Tailandia. UNESCO.

UNESCO. (1994). Conferencia Mundial Sobre Necesidades Educativas Especiales: Acceso y Calidad. Salamanca, España.

Vega, A. (2001). La educación de los niños con síndrome de Down. Principios y prácticas. Salamanca, España: Editorial Amarú. 